\title{
Intracranial Aneurysms: Wall Motion Analysis for Prediction of Rupture
}

\author{
(D)A.E. Vanrossomme, O.F. Eker, J.-P. Thiran, G.P. Courbebaisse, and K. Zouaoui Boudjeltia
}

\begin{abstract}
SUMMARY: Intracranial aneurysms are a common pathologic condition with a potential severe complication: rupture. Effective treatment options exist, neurosurgical clipping and endovascular techniques, but guidelines for treatment are unclear and focus mainly on patient age, aneurysm size, and localization. New criteria to define the risk of rupture are needed to refine these guidelines. One potential candidate is aneurysm wall motion, known to be associated with rupture but difficult to detect and quantify. We review what is known about the association between aneurysm wall motion and rupture, which structural changes may explain wall motion patterns, and available imaging techniques able to analyze wall motion.
\end{abstract}

$I^{\mathrm{n}}$ ntracranial aneurysms are abnormal focal dilations of the cerebral vasculature with a prevalence approximating $2 \%-5 \% .^{1-3}$ Complications associated with intracranial aneurysms include mass effect on adjacent structures - cranial nerves, brain stem, and so forth ${ }^{4,5}$ - and rupture, the most severe and frequent complication. The estimated incidence of this latter complication is approximately $1 \%$ per aneurysm and per year. ${ }^{3,6}$ With short-term mortality from rupture ranging from $35 \%$ to $50 \%$ and with almost half of survivors having moderate-to-severe long-term disability, ${ }^{6-8}$ neurosurgical and endovascular treatment could potentially be advocated for all unruptured intracranial aneurysms. However, these treatments are associated with approximate mortality rates of $3 \%$ and $2 \%$ and long-term disability rates of $15 \%$ and $5 \%,{ }^{8-10}$ respectively, so their use remains controversial. Current guidelines for recommending treatment are based on aneu-

From the Laboratory of Experimental Medicine (A.E.V., K.Z.B.), Université Libre de Bruxelles, Bruxelles, Belgium; Department of Interventional Neuroradiology (O.F.E.), Gui de Chauillac Hospital, Centre Hospitalier Régional Universitaire Montpellier, Montpellier, France; Signal Processing Laboratory (J.-P.T.), Swiss Federal Institute of Technology Lausanne, Lausanne, Switzerland; Department of Radiology (J.-P.T.), University Hospital Center and University of Lausanne, Lausanne, Switzerland; and Centre de Recherche en Acquisition et Traitement de l'Image pour la Santé - Centre National de Recherche Scientifique - Unité Mixte de Recherche 5220 (G.P.C.), Institut National des Sciences Appliquées Lyon, Université de Lyon, Lyon, France.

A.E.V. is supported by the Fonds Erasme pour la Recherche Médicale, Université Libre de Bruxelles, Brussels, Belgium. This research received funding from the Thrombus Project (European Commission, 7th Framework Project) under grant agreement reference 269966

Please address correspondence to Axel Vanrossomme, MD, Laboratoire de Médecine Expérimentale, Centre Hospitalier Universitaire André Vésale, Rue de Gozée 706, 6110 Montigny-le-Tilleul, Belgium; e-mail: avrossom@ulb.ac.be

- Indicates open access to non-subscribers at www.ajnr.org

http://dx.doi.org/10.3174/ajnr.A4310

rysm size, location, and patient age. ${ }^{11,12}$ The Unruptured Cerebral Aneurysm Study suggests treating small aneurysms in locations associated with high risk of rupture and observing larger ones, up to $10 \mathrm{~mm}$, in locations associated with a low risk of rupture (internal carotid, middle cerebral arteries). ${ }^{6}$ The Population, Hypertension, Age, Size of Aneurysm, Earlier Subarachnoid Hemorrhage from Another Aneurysm, and Site of Aneurysm (PHASES) score allows the calculation of a 5-year rupture risk, depending on multiple factors. ${ }^{13}$ This score allows a better assessment of the benefits versus risks of treatment in daily practice. However, for aneurysms considered at low risk of rupture, additional criteria are needed to discriminate those that may rupture and therefore should be treated and those that just require surveillance.

Aneurysm wall motion has been suggested to potentially provide predictive information on the risk of rupture. Numerous studies reported the detection of wall motion through different imaging techniques, such as phase-contrast MRA, transcranial power Doppler ultrasonography, 4D-CTA, and $3 \mathrm{D}$ rotational angiography (Table). ${ }^{14-33}$ Among these studies, some have reported an association between rupture status and wall motion.

Structurally speaking, the heterogeneous thickness of the aneurysm wall and its infiltration by inflammatory cells have also been reported ${ }^{34}$; whether and how these factors can modify wall motion is not understood.

This article reviews the current knowledge of the association between aneurysm wall motion and rupture, the structural changes that could explain the wall motion patterns, and imaging techniques able to analyze wall motion. 


\begin{tabular}{|c|c|c|c|c|c|c|}
\hline $\begin{array}{l}\text { Authors and Year } \\
\text { of Publication }\end{array}$ & Imaging Modality & Study Object & No. & $\begin{array}{l}\text { Aneurysms and } \\
\text { Rupture Status }\end{array}$ & $\begin{array}{l}\text { Frequency of Wall } \\
\text { Motion Detection }\end{array}$ & $\begin{array}{l}\text { Wall Motion } \\
\text { Quantification }\end{array}$ \\
\hline Wardlaw and Cannon (1996) & PD-US & In vivo & 40 & $6 U / 31 R$ & Not reported & Yes \\
\hline Wardlaw et al (1998) & PD-US & In vivo & 9 & $3 U / 9 R$ & Not reported & Yes \\
\hline Hoskins et al $(1998)^{16}$ & PD-US & Phantom & NA & NA & NA & Yes \\
\hline Kato et al $(2004)^{17}$ & 4D-CTA & In vivo & 15 & $15 U$ & $10 / 15^{\mathrm{a}}$ & No \\
\hline Hayakawa et al $(2005)^{18}$ & 4D-CTA & In vivo & 23 & $23 R$ & $4 / 23$ & No \\
\hline Ishida et al $(2005)^{19}$ & 4D-CTA & In vivo & 30 & $29 U / 5 R$ & $13 / 34$ & No \\
\hline Yaghmai et al $(2007)^{20}$ & 4D-CTA & Phantom & NA & NA & NA & No \\
\hline Krings et al $(2009)^{21}$ & 4D-CTA & In vivo & 1 & $1 \mathrm{U}$ & $1 / 1$ & Yes \\
\hline Hayakawa et al (2011) ${ }^{22}$ & 4D-CTA & In vivo & 51 & $53 \mathrm{U} / 12 \mathrm{R}$ & $24 / 65$ & No \\
\hline Kuroda et al $(2012)^{23}$ & 4D-CTA & In vivo & 18 & $22 \mathrm{U}$ & Not reported & Yes \\
\hline Firouzian et al $(2013)^{24}$ & 4D-CTA & In vivo & 14 & $19 U$ & $19 / 19$ & Yes \\
\hline Hayakawa et al $(2014)^{25}$ & 4D-CTA & In vivo & 37 & $56 U$ & $20 / 56$ & No \\
\hline Meyer et al $(1993)^{26}$ & PC-MRA & In vivo & 15 & $10 U / 6 R$ & $15 / 16$ & Yes \\
\hline Karmonik et al $(2010)^{27}$ & PC-MRA & In vivo & 7 & $7 U$ & $7 / 7$ & Yes \\
\hline Dempere-Marco et al $(2006)^{28}$ & 3D RA & In vivo & 3 & $3 U$ & $2 / 3$ & Yes \\
\hline Oubel et al $(2007)^{29}$ & 3D RA & In vivo & 4 & $4 U$ & $4 / 4$ & Yes \\
\hline Zhang et al $(2009)^{30}$ & 3D RA & Phantom & NA & NA & NA & Yes \\
\hline Zhang et al (2009) ${ }^{31}$ & 3D RA & Phantom & NA & NA & NA & Yes \\
\hline Oubel et al $(2010)^{32}$ & 3D RA & In vivo & 18 & $11 U / 7 R$ & $10 / 18$ & Yes \\
\hline Zhang et al (2011) & 3D RA & Phantom + in vivo & 2 & $2 U$ & $1 / 2$ & Yes \\
\hline
\end{tabular}

Note:-No. indicates number of patients; PD-US, power Doppler ultrasonography; PC-MRA, phase-contrast MR angiography; 3D RA, 3D rotational angiography; U, unruptured; $\mathrm{R}$, ruptured; NA, not applicable.

authors focused on bleb detection only. Pulsating bleb was found in 10 of the 15 cases.

\section{ASSOCIATION BETWEEN WALL MOTION AND RUPTURE}

Meyer et $\mathrm{al}^{26}$ first suggested a possible association between wall motion patterns and aneurysm rupture. These authors retrospectively compared the flow dynamics of 6 ruptured and 10 unruptured aneurysms by using cine phase-contrast MRA. They observed that the volume of ruptured aneurysms increased more from diastole to systole than did the volume of unruptured aneurysms $(51.0 \pm 10.0 \%$ versus $17.6 \pm 8.9 \%, P<.005)$, independent of aneurysm size. This study, however, is limited in its conclusions. First, it is outdated, because MR imaging has improved considerably during these past 2 decades, and cine phase-contrast MRA would not be the technique of choice today. Moreover the postprocessing technique is also outdated with regard to current standards. Second, phase-contrast techniques are prone to flow artifacts and might, therefore, mistake slow flow for wall motion. Third, this study was based on a small number of patients and was retrospective with regard to rupture status. Therefore, caution is needed in the interpretation of its results.

Using 4D-CTA, Hayakawa et al ${ }^{18}$ observed aneurysm wall motion in 4 of 23 patients with ruptured aneurysms. During surgical clipping performed in 2 of these 4 patients, they observed that the rupture site matched the position of the wall motion detected by 4D-CTA. Ishida et al ${ }^{19}$ studied 30 patients by using the same $4 \mathrm{D}-$ CTA technique and observed a pulsating bleb in 7 of 29 unruptured aneurysms and in 2 of 5 ruptured ones. Again, during surgical clipping performed in these 2 ruptured aneurysms, these authors observed that the rupture site matched the position of the pulsating bleb. These 2 studies are interesting but focused on the detection of blebs and their motion. They showed some ability to detect the rupture site when blebs ruptured but did not investigate the relation between motion and rupture. Other limitations include the following: the limited number of patients, especially in the ruptured group of the study of Ishida et $\mathrm{al}^{19}$; the subjective assessment of the rupture point by the neurosurgeon not relying on precise spatial correlation; and apparent motion artifacts of immobile bone structures, possibly due to gantry rotation as emphasized by Matsumoto et $\mathrm{al}^{35}$ in a letter to the authors. Ishida et al replied that more work was needed to validate 4D-CTA for aneurysm dynamics visualization and that this work was just a step along the path.

Oubel et $\mathrm{al}^{32}$ introduced the concept of differential pulsation, defined as the aneurysm wall motion corrected for the wall motion of the parent artery and its diameter. Using high-frame-rate DSA, these authors measured aneurysm wall motion in 18 patients with 7 ruptured aneurysms and reported a statistically significant association between this differential pulsation and the rupture status. Kuroda et $\mathrm{al}^{23}$ found no significant difference in cardiac cycle-related volume changes between unruptured aneurysms and normal arteries assessed by 4D-CTA in 18 patients, suggesting that the differential pulsation may be a more useful indicator than the wall motion magnitude or the cardiac cyclerelated volume changes to discriminate aneurysms at high-versus-low risk of rupture.

These studies suggest a possible association between aneurysm wall motion and rupture, but they share 2 limitations: the small numbers of patients studied and the aneurysms being considered retrospectively after rupture instead of prospectively considering those likely to rupture. A recent study indeed showed that aneurysm characteristics can be different before and after rupture and suggests caution with the interpretation of such results. ${ }^{36}$ These limitations support the need for a larger study, prospective with regard to rupture status, to test whether the outcome (rupture, growth of the aneurysm, and so forth) depends on the type of wall motion. Ideally, unruptured aneurysms should be followed without treatment until they rupture, but this plan would be unethical except with patients who refuse treatment.

AJNR Am J Neuroradiol 36:1796-802 Oct 2015 www.ajnr.org 


\section{ASSOCIATION BETWEEN ANEURYSM WALL CHANGES AND RUPTURE}

The differences in wall motion between ruptured and unruptured aneurysms suggest differences in wall architecture or composition, including inflammatory and atherosclerotic changes and structural matrix proteins.

\section{Wall Architecture}

The aneurysm wall is histologically characterized by a loss or disruption of internal elastic lamina, intimal hyperplasia, disorganization of smooth-muscle fibers, loss of cell components, and infiltration by inflammatory cells. ${ }^{34,37-41}$ Disruption of the internal elastic lamina seems to occur early during aneurysm formation, ${ }^{42-46}$ whereas other changes (eg, de-endothelialization, luminal thrombosis, smooth-muscle cell proliferation, T-cell and macrophage infiltration) are associated with rupture. ${ }^{34}$ Frösen et $\mathrm{al}^{34}$ identified 4 wall types associated with rupture: type A with an endothelialized wall with linearly organized smooth-muscle cells; type B with a thickened wall with disorganized smoothmuscle cells; type $\mathrm{C}$ with a hypocellular wall with either intimal hyperplasia or organizing luminal thrombus; and type D with an extremely thin, thrombosis-lined hypocellular wall. The rupture rate increased from type A to D. These authors suggested that these 4 types are most likely consecutive stages of wall degeneration leading to rupture. In addition, there was usually a type Ato- $\mathrm{D}$ gradient from the neck to the fundus. ${ }^{34}$ This illustrates the heterogeneity of the wall, which can be seen as a global vascular disease with focal progression leading to rupture. Whether these wall types relate to wall motion modification is not fully understood. However, Costalat et $\mathrm{al}^{47}$ described 3 kinds of aneurysm wall biomechanical properties-rigid, intermediate, and softthe latter was only seen in ruptured or preruptured aneurysms. This latter study supports the hypothesis of a motion of greater magnitude in ruptured and preruptured aneurysms. Moreover, the elastic modulus at the aneurysm fundus being 30\%-50\% lower than that at the neck has been described; the fundus wall is therefore more distensible. ${ }^{48}$

\section{Inflammatory and Atherosclerotic Changes in the Aneurysm Wall}

Inflammatory changes in the aneurysm wall are characterized by leukocyte infiltration, leading to smooth-muscle cell proliferation and fibrosis. ${ }^{34,39}$ Smooth-muscle cells and macrophages produce matrix metalloproteinases, mostly matrix metalloproteinase-1, -2 , and -9 , which degrade extracellular matrix components. Early on, matrix metalloproteinases are balanced by tissue inhibitor of metalloproteinase-1 and -2, but not in late-stage aneurysms, in which the expression of matrix metalloproteinases is increased; this change leads to extracellular matrix damage, growth of the aneurysm, and rupture. ${ }^{49-51}$

Atherosclerotic changes seem to be present in most aneurysms, and their extent correlates with aneurysm growth. Small aneurysms have atherosclerotic lesions characterized by intimal thickening due to proliferating smooth-muscle cells with few macrophages and lymphocytes, whereas large aneurysms have more advanced lesions with more cellular infiltrates and mature smooth-muscle cells. ${ }^{52,53}$ In addition, aneurysm smooth-muscle cells acquire a dedifferentiated phenotype similar to that in smooth-muscle cells in atherosclerotic plaques. ${ }^{54}$ Most interesting, atherosclerosis is associated with chronic inflammation, which might explain, at least in part, the inflammatory changes. ${ }^{55-57}$

\section{Structural Matrix Proteins}

Collagen. Collagen content is lower in aneurysm walls than in normal arteries. In addition, the number of cross-links between collagen fibers is lower, and collagenase and elastase activities are higher in ruptured aneurysms than in unruptured ones. These comparisons raise the hypothesis that elevated collagenolytic and elastolytic activities could be predisposing factors for rupture. ${ }^{58}$ Additionally, while expression of collagen III and IV is lower in all (unruptured and ruptured) aneurysms than in normal arteries, collagen IV expression is lower in ruptured aneurysms than in unruptured ones. ${ }^{38}$

Laminin and Fibronectin. Laminin and fibronectin are thought to maintain the structural integrity of the vessel wall by anchoring endothelial cells to the internal elastic lamina and smooth-muscle layers. Laminin is more cohesive than fibronectin and is predominant in mature vessels, whereas fibronectin is predominant in immature ones. While the expression of laminin is lower in both unruptured and ruptured aneurysms than in normal arteries, the expression of fibronectin is only higher in ruptured aneurysms, raising the hypothesis that an increased fibronectin-to-laminin ratio might contribute to rupture. ${ }^{38}$

Alpha-Smooth-Muscle Actin. Alpha-smooth-muscle actin expression is lower in aneurysm walls than in normal arteries and even lower in ruptured aneurysms than in unruptured ones. In addition, and in contrast to normal arteries and unruptured aneurysms, the myocytes in ruptured aneurysms are loose and no longer arranged in tightly compacted functional bands. ${ }^{38}$

Changes in structural matrix proteins raise the hypothesis that collagen III, collagen IV, and $\alpha$-smooth-muscle actin are involved in aneurysm formation and that an increase in the fibronectin-tolaminin ratio could contribute to rupture. Because the expression of collagen IV and laminin is maximal in mature vessels whereas fibronectin is usually found in immature ones, aneurysm formation and rupture seem to be associated with an angiogenically immature vessel wall. ${ }^{38}$ It was hypothesized that an increase in immature collagen, lower collagen content, or a less structured wall could lead to a more distensible behavior. ${ }^{48}$

\section{IMAGING ANEURYSM WALL MOTION}

The imaging of wall motion has multiple challenges. First, the motion itself is 3-fold: the global pulsation of the aneurysm, potential movements of focal parts of the wall (eg, blebs), and global cerebral vasculature motion during the cardiac cycle. ${ }^{18,19,59,60}$ Second, the magnitude of motion is small, and the timeframe is short; thus, techniques with high spatial and temporal resolutions are needed. Third, depending on the technique, artifacts can impair the results and they must be kept to a minimum. The radiation dose must also be considered when using dynamic $\mathrm{x}$-raybased imaging modalities. Six imaging techniques can detect aneurysm wall motion, but only 4 (Table) are used in living humans. The other 2 are based on laser displacement sensor and flat 
panel volumetric CT. Laser displacement sensor has been used to detect aneurysm wall motion of an ex vivo animal model and a silicone phantom in 2 studies. ${ }^{61,62}$ Flat panel volumetric CT has been used to image posttreatment rabbit aneurysm pulsation, but though promising, this tool has never been used to image aneurysm wall motion in humans. ${ }^{63,64}$

The 4 techniques applied in living humans are transcranial power Doppler ultrasonography, phase-contrast MRA, 3D rotational angiography, and 4D-CTA.

\section{Transcranial Power Doppler Ultrasonography}

Transcranial power Doppler ultrasonography was developed as a tool to image blood flow in intracranial vessels. With a higher sensitivity to blood flow than color Doppler, power Doppler is more appropriate for small-vessel imaging. This technique was used by the same group of investigators to detect aneurysm pulsation in 2 in vivo studies and 1 in vitro study. The pulsation was quantified by comparing the aneurysm cross-sectional area between systole and diastole, measured by using the sonography device algorithm. ${ }^{14-16}$ Aneurysm pulsation was detected in both in vivo studies, but its magnitude was much larger than that usually seen with other techniques and during neurosurgery. The in vitro study revealed that pulsation as seen on power Doppler depended on the device settings, introducing dependency on the operator and raising concerns about its reproducibility. ${ }^{16}$ Moreover, some of the observed pulsation may be related to very slow intra-aneurysmal blood flow during part of the cardiac cycle, leading to the absence of a Doppler signal and hence exaggerating apparent wall motion.

Other limitations of transcranial power Doppler ultrasonography are limited acoustic windows due to bone structures; additionally, $20 \%$ of patients have poor or absent acoustic windows ${ }^{65}$ and limited spatial resolution, especially with low-frequency transducers that are required to image deep arteries. Temporal and spatial resolutions are indeed dependent on the frequency of the transducer and the depth of the structure of interest. Axial resolution is half the spatial pulse length, which is the product of the number of cycles in a pulse and the wavelength. Lateral resolution depends on the wavelength and the width of the transducer and can be improved by focusing ultrasounds; temporal resolution of Doppler depends on the pulse length, the depth of the structure of interest, and the size of the color box. ${ }^{66}$ Since these studies were published, progress has been made in transcranial Doppler imaging, which now shows good agreement with CTA as demonstrated for intracranial vessel localization. ${ }^{67}$ Transcranial power Doppler ultrasonography could, therefore, be re-evaluated for intracranial aneurysm imaging.

\section{Cine Phase-Contrast MRA}

Phase-contrast MRA is based on the measurement of the phase shift of blood flowing through a magnetic field gradient; the sign of the phase shift is determined by the direction of the flow. Because phase-contrast sequences are only sensitive to a given range of blood velocities that must be determined a priori, the optimal sequence must be tuned for each patient. Nevertheless, even with optimal velocity-encoding, the signal within the aneurysm could be impaired because of the highly heterogeneous intrasaccular flow. Although this technique has the advantage of not exposing the patient to ionizing radiation or contrast material, it is limited by a low sensitivity for motion depiction. Coupled with gadolinium-enhanced MRA, this technique provides information on morphologic and flow characteristics, while the temporal resolution of MRA alone is insufficient to assess aneurysm wall motion. ${ }^{68}$ Two studies showed that aneurysm wall motion could be detected and quantified with this technique. Quantification of motion was achieved either by volume quantification for each phase ${ }^{26}$ or by measuring the distance between the aneurysm wall and the center of the aneurysm to avoid taking global artery motion into account. ${ }^{27}$ Meyer et $\mathrm{al}^{26}$ showed that volume modification during the cardiac cycle was larger in ruptured aneurysms than in unruptured ones. Focusing on unruptured aneurysms, Karmonik et $\mathrm{al}^{27}$ showed that in addition to outward and inward wall displacement during the cardiac circle, there was local heterogeneity within the aneurysm wall, consisting of out-of-phase movements of some parts compared with others.

Phase-contrast MRA has several limitations. First, because of the lack of standardized blood-velocity values, acquisition time is too long for clinical use. Second, its spatial resolution is low but could be improved with high-field MR imaging scanners. ${ }^{27}$ Third, motion (of cerebral arteries during the cardiac cycle or of the patient) and flow artifacts from out-of-range velocities can decrease image quality. The current best techniques are probably conventional gradient echo-based cine imaging sequences (eg, gradient-recalled acquisition in steady state [GRASS], true fast imaging with steady-state precession [trueFISP], true spin tagging with alternating radiofrequency [trueSTAR]) ${ }^{69,70}$ These methods have high spatial resolution (up to $1 \mathrm{~mm}^{3}$ voxels or $0.86 \times 0.86 \times 2 \mathrm{~mm}^{3}$ voxels) and high temporal resolution (50$100)$ and have a higher SNR than phase-contrast sequences, making them promising tools to image wall motion. Moreover, increasing the magnetic field strength from $1.5 \mathrm{~T}$ to $3 \mathrm{~T}$ or using a gadolinium-based contrast agent could enhance SNR as reported for cardiac investigations. ${ }^{71}$ Usual dynamic contrast-enhanced MRA techniques that rely on parallel imaging, partial $k$-space sampling, or half-Fourier would not be appropriate for wall motion imaging because the temporal resolutions are not sufficient $(500-1500 \mathrm{~ms}) .^{72}$

\section{D Rotational Angiography}

$3 \mathrm{D}$ rotational angiography is an $\mathrm{x}$-ray-based projection imaging technique in which both the source and the detector rotate around approximately $200^{\circ}$. In a clinical setting, this technique is based on acquisition of a sequence of $2 \mathrm{D}$ images that are used to reconstruct an isotropic $3 \mathrm{D}$ image, but $2 \mathrm{D}$ images can also be used to retrieve dynamic information and detect wall motion. Using this technique, several authors succeeded in detecting and quantifying wall motion. ${ }^{28-33}$ Quantification was achieved through either an optical flow method for image registration or, later, freeform deformations with B-spline interpolation. ${ }^{29,31,32}$ In 3 articles reporting the data from 18 patients, Dempere-Marco et $\mathrm{al}^{28}$ and Oubel et al, ${ }^{29,32}$ demonstrated the ability to detect and quantify wall motion with this technique. During the same period, Zhang et $\mathrm{al}^{30,31,33}$ applied this technique on in silico models,

AJNR Am J Neuroradiol 36:1796-802 Oct 2015 www.ajnr.org

1799 
on in vitro samples, and in vivo, but only in 2 patients. This technique has high spatial and temporal resolutions (respectively, 0.2 $\mathrm{mm}$ and from 35 to $165 \mathrm{~ms}$, depending on vessel configuration). ${ }^{73}$ However, this technique is invasive, exposes the patient to ionizing radiation, and requires injection of iodinated contrast material. It is also challenging to ensure homogeneous contrast distribution inside the aneurysm during the acquisition. Because 3D rotational angiography is the most invasive technique among the 4 discussed in this review, it should probably be reserved for a subset of selected patients that still needs to be defined (eg, patients already programmed for a catheter-injected angiography could benefit from this dynamic technique).

\section{D-CTA}

Faster gantry rotation and multidetector rows are major developments in CT technology, which have led to increased spatial and temporal resolutions. In addition, with an effective scan width of $>20$ and $\leq 160 \mathrm{~mm}$, scanners with $\geq 64$ detector rows allow continuous acquisition without table movement and subsequent $4 \mathrm{D}$ reconstructions. Several studies used $4 \mathrm{D}-\mathrm{CTA}$ and reported successful detection of aneurysm wall motion in phantoms and in patients. Although most studies were based on electrocardiogram-gated 4D-CTA, ${ }^{17-19,22-25}$ some reported that pulsatility imaging was also possible without gating. ${ }^{20,21}$ The analysis of wall motion was either qualitative with visual assessment by multiple readers or quantitative. For quantitative analysis, some authors chose to convert the original matrix into an easier-to-process "black and white" matrix. Hounsfield units that were out of a predetermined range (approximately 100-900 HU) were substituted for zero, whereas Hounsfield units within the range were substituted for 1 . This matrix could then be analyzed through segmentation of the aneurysm sac and the calculation of the aneurysm volume for each frame. ${ }^{23}$ Other authors used nonrigid B-spline registration to obtain a motion-compensated multiphase $3 \mathrm{D}$ image and a deformation field for each phase. The deformation field could then be combined with aneurysm segmentation to determine wall deformation. ${ }^{24}$ Two studies investigated the motion of cerebral arteries and showed their 2-type motion: pulsation consisting of changes in vessel volume and a positional change of the whole vessel during the cardiac cycle. ${ }^{59,60}$ Moreover, the direction and the amplitude of these movements differed from one artery to another in the same subject. ${ }^{59}$

4D-CTA has several advantages. First, scanners are available in almost all hospitals in developed countries. Second, the duration of the examination is very short because its acquisition lasts just a few seconds. Third, while of the same order of magnitude as the analyzed motion, its spatial resolution is high $(0.25 \mathrm{~mm}$ for highresolution CT scans and from 0.60 to $0.80 \mathrm{~mm}$ for standard CT scans) $)^{74}$ and its temporal resolution ranges between 75 and 150 ms. ${ }^{75}$ Nevertheless, this technique has limitations. First, although rarely addressed in the clinical studies discussed here, the radiation dose for dynamic acquisitions can be very high. Second, intravenous iodine contrast injection is needed. Third, images can be impaired by reconstruction artifacts that are reduced with multidetector row CT scanners.

To reduce these reconstruction artifacts, one could analyze wall motion by using the raw data (ie, the sinogram). The registration technique used with $3 \mathrm{D}$ rotational angiography is extended and adapted to 4D-CTA. ${ }^{32,33}$ Using the sinogram instead of reconstructed images would improve both spatial and temporal resolution appropriate for detecting small-amplitude motion. ${ }^{76,77}$

\section{CONCLUSIONS}

Guidelines for treatment of intracranial aneurysms need to include better tools to appropriately predict aneurysm rupture. The PHASES score provides useful information about the outcome, but other tools might help refine the rupture risk. The analysis of aneurysm wall motion seems promising for this purpose. Multiple retrospective clinical studies have shown an association between wall motion and rupture status, but there is a lack of prospective studies to assess this relationship. The hypothesis that a difference in wall motion patterns is associated with an increased risk of rupture is also theoretically supported by the demonstration of histologic alterations of the aneurysm wall, most notably thinning and loss of mural cells in the most advanced stages leading to a more distensible behavior predominantly at the fundus. This could explain why the largest motion amplitude is usually seen at the fundus. Up to now, no study has investigated the relationship between histologic changes and biomechanical properties of the wall.

With regard to imaging modalities, there is no consensus on the best available technique, but $3 \mathrm{D}$ rotational angiography and $4 \mathrm{D}$-CTA seem the most promising because they are the least subject to flow artifacts; however, the radiation dose should not be neglected, especially in young patients. ${ }^{78}$ For patients who need conventional angiography anyway, dynamic 3D rotational angiography would be the best available technique. Finally, the lack of standardization in imaging protocols and reconstruction algorithms should be resolved to ensure that research data are comparable.

\section{ACKNOWLEDGMENTS}

The authors thank Pierre Alain Gevenois, $\mathrm{MD}, \mathrm{PhD}$, for his invaluable assistance in preparing the manuscript and Nathalie Nagy, MD, for her valuable advice on the histology of intracranial aneurysms.

Disclosures: Axel Vanrossome-RELATED: Grant: My research activities are supported entirely by the "Fonds Erasme pour le recherche médicale" from October 2013 to September 2015. Jean-Philippe Thiran—RELATED: Grant: European Unionfunded Thrombus Project financed this work. ${ }^{*}$ Karim Zouaoui Boudjeltia—RELATED: Grant: European PF7 Grant: Thrombus Project.* *Money paid to the institution.

\section{REFERENCES}

1. Vlak MH, Algra A, Brandenburg R, et al. Prevalence of unruptured intracranial aneurysms, with emphasis on sex, age, comorbidity, country, and time period: a systematic review and meta-analysis. Lancet Neurol 2011;10:626-36

2. Vega C, Kwoon JV, Lavine SD. Intracranial aneurysms: current evidence and clinical practice. Am Fam Physician 2002;66:601-08

3. Rinkel GJ, Djibuti M, Algra A, et al. Prevalence and risk of rupture of intracranial aneurysms: a systematic review. Stroke 1998;29:251-56

4. Hassan T, Hamimi A. Successful endovascular management of brain aneurysms presenting with mass effect and cranial nerve palsy. Neurosurg Rev 2013;36:87-97; discussion 97

5. van Rooij WJ, Sluzewski M. Unruptured large and giant carotid ar- 
tery aneurysms presenting with cranial nerve palsy: comparison of clinical recovery after selective aneurysm coiling and therapeutic carotid artery occlusion. AJNR Am J Neuroradiol 2008;29:997-1002

6. Morita A, Kirino T, Hashi K, et al. The natural course of unruptured cerebral aneurysms in a Japanese cohort. $N$ Engl J Med 2012;366: 2474-82

7. Morita A, Fujiwara S, Hashi K, et al. Risk of rupture associated with intact cerebral aneurysms in the Japanese population: a systematic review of the literature from Japan. J Neurosurg 2005;102:601-06

8. International Study of Unruptured Intracranial Aneurysms Investigators. Unruptured intracranial aneurysms: risk of rupture and risks of surgical intervention. $N$ Engl J Med 1998;339:1725-33

9. Pierot L, Barbe C, Spelle L; ATENA investigators. Endovascular treatment of very small unruptured aneurysms: rate of procedural complications, clinical outcome, and anatomical results. Stroke 2010;41:2855-59

10. McDonald JS, McDonald RJ, Fan J, et al. Comparative effectiveness of unruptured cerebral aneurysm therapies: propensity score analysis of clipping versus coiling. Stroke 2013;44:988-94

11. Komotar RJ, Mocco J, Solomon RA. Guidelines for the surgical treatment of unruptured intracranial aneurysms: the first annual J Lawrence Pool Memorial Research Symposium-controversies in the management of cerebral aneurysms. Neurosurgery 2008;62:18393; discussion 193-94

12. Steiner T, Juvela S, Unterberg A, et al; European Stroke Organization. European Stroke Organization guidelines for the management of intracranial aneurysms and subarachnoid haemorrhage. Cerebrovasc Dis 2013;35:93-112

13. Greving JP, Wermer MJ, Brown RD Jr, et al. Development of the PHASES score for prediction of risk of rupture of intracranial aneurysms: a pooled analysis of six prospective cohort studies. Lancet Neurol 2014;13:59-66

14. Wardlaw JM, Cannon JC. Color transcranial "power" Doppler ultrasound of intracranial aneurysms. J Neurosurg 1996;84:459-61

15. Wardlaw JM, Cannon J, Statham PF, et al. Does the size of intracranial aneurysms change with intracranial pressure? Observations based on color "power" transcranial Doppler ultrasound. J Neurosurg 1998;88:846-50

16. Hoskins PR, Prattis J, Wardlaw J. A flow model of cerebral aneurysms for use with power Doppler studies. $\mathrm{Br} J$ Radiol 1998;71:76-80

17. Kato $Y$, Hayakawa $M$, Sano $H$, et al. Prediction of impending rupture in aneurysms using 4D-CTA: histopathological verification of a real-time minimally invasive tool in unruptured aneurysms. Minim Invasive Neurosurg 2004;47:131-35

18. Hayakawa M, Katada K, Anno H, et al. CT angiography with electrocardiographically gated reconstruction for visualizing pulsation of intracranial aneurysms: identification of aneurysmal protuberance presumably associated with wall thinning. AJNR Am J Neuroradiol 2005;26:1366-69

19. Ishida F, Ogawa H, Simizu T, et al. Visualizing the dynamics of cerebral aneurysms with four-dimensional computed tomographic angiography. Neurosurgery 2005;57:460-71; discussion 460-71

20. Yaghmai V, Rohany M, Shaibani A, et al. Pulsatility imaging of saccular aneurysm model by 64-slice CT with dynamic multiscan technique. J Vasc Interv Radiol 2007;18:785-88

21. Krings $T$, Willems $P$, Barfett $J$, et al. Pulsatility of an intracavernous aneurysm demonstrated by dynamic 320-detector row CTA at high temporal resolution. Cent Eur Neurosurg 2009;70:214-18

22. Hayakawa M, Maeda S, Sadato A, et al. Detection of pulsation in ruptured and unruptured cerebral aneurysms by electrocardiographically gated 3-dimensional computed tomographic angiography with a 320-row area detector computed tomography and evaluation of its clinical usefulness. Neurosurgery 2011;69:843-51; discussion 851

23. Kuroda J, Kinoshita $\mathrm{M}$, Tanaka $\mathrm{H}$, et al. Cardiac cycle-related volume change in unruptured cerebral aneurysms: a detailed volume quantification study using 4-dimensional CT angiography. Stroke 2012;43:61-66

24. Firouzian A, Manniesing R, Metz CT, et al. Quantification of intracranial aneurysm morphodynamics from ECG-gated CT angiography. Acad Radiol 2013;20:52-58

25. Hayakawa M, Tanaka T, Sadato A, et al. Detection of pulsation in unruptured cerebral aneurysms by ECG-gated 3D-CT angiography (4D-CTA) with 320-row area detector CT (ADCT) and follow-up evaluation results: assessment based on heart rate at the time of scanning. Clin Neuroradiol 2014;24:145-50

26. Meyer FB, Huston J3rd, Riederer SS. Pulsatile increases in aneurysm size determined by cine phase-contrast MR angiography. J Neurosurg 1993;78:879-83

27. Karmonik C, Diaz O, Grossman R, et al. In-vivo quantification of wall motion in cerebral aneurysms from 2D cine phase contrast magnetic resonance images. Rofo 2010;182:140-50

28. Dempere-Marco L, Oubel E, Castro M, et al. CFD analysis incorporating the influence of wall motion: application to intracranial aneurysms. In: Proceedings of the 9th International Conference of the Medical Image Computing and Computer-Assisted Intervention Society, Copenhagen, Denmark. October 1-6, 2006

29. Oubel E, De Craene M, Putman CM, et al. Analysis of intracranial aneurysm wall motion and its effects on hemodynamic patterns. In: Proceedings of the Society of Photo-Optical Instrumentation Engineers, Medical Imaging: Physiology, Function, and Structure from Medical Images, San Diego, California. February 17-22, 2007

30. Zhang C, De Craene M, Villa-Uriol MC, et al. Estimating continuous $4 \mathrm{D}$ wall motion of cerebral aneurysms from $3 \mathrm{D}$ rotational angiography. In: Proceedings of the 12th International Conference of the Medical Image Computing and Computer-Assisted Society, London, United Kingdom. September 20-24, 2009

31. Zhang C, Villa-Uriol MC, De Craene M, et al. Morphodynamic analysis of cerebral aneurysm pulsation from time-resolved rotational angiography. IEEE Trans Med Imaging 2009;28:1105-16

32. Oubel E, Cebral JR, De Craene M, et al. Wall motion estimation in intracranial aneurysms. Physiol Meas 2010;31:1119-35

33. Zhang C, Villa-Uriol MC, De Craene M, et al. Dynamic estimation of three-dimensional cerebrovascular deformation from rotational angiography. Med Phys 2011;38:1294-306

34. Frösen J, Piippo A, Paetau A, et al. Remodeling of saccular cerebral artery aneurysm wall is associated with rupture: histological analysis of 24 unruptured and 42 ruptured cases. Stroke 2004;35:2287-93

35. Matsumoto M, Sasaki T, Suzuki K, et al. Visualizing the dynamics of cerebral aneurysms with four-dimensional computed tomographic angiography. Neurosurgery 2006;58:E1003; author reply E1003

36. Schneiders JJ, Marquering HA, van den Berg R, et al. Rupture-associated changes of cerebral aneurysm geometry: high-resolution 3D imaging before and after rupture. AJNR Am J Neuroradiol 2014;35:1358-62

37. Abruzzo T, Shengelaia GG, Dawson RC 3rd, et al. Histologic and morphologic comparison of experimental aneurysms with human intracranial aneurysms. AJNR Am J Neuroradiol 1998;19:1309-14

38. Kilic T, Sohrabifar M, Kurtkaya O, et al. Expression of structural proteins and angiogenic factors in normal arterial and unruptured and ruptured aneurysm walls. Neurosurgery 2005;57:997-1007; discussion 997-1007

39. Chyatte D, Bruno G, Desai S, et al. Inflammation and intracranial aneurysms. Neurosurgery 1999;45:1137-46; discussion 1146-47

40. Scanarini M, Mingrino S, Giordano R, et al. Histological and ultrastructural study of intracranial saccular aneurysmal wall. Acta Neurochir (Wien) 1978;43:171-82

41. Schlote W, Gaus C. Histologic aspects from ruptured and nonruptured aneurysms. Neurol Res 1994;16:59-62

42. Kim C, Cervós-Navarro J, Kikuchi H, et al. Degenerative changes in the internal elastic lamina relating to the development of saccular cerebral aneurysms in rats. Acta Neurochir (Wien) 1993;121:76-81

43. Kim C, Kikuchi H, Hashimoto N, et al. Involvement of internal elas- 
tic lamina in development of induced cerebral aneurysms in rats. Stroke 1988;19:507-11

44. Hazama F, Hashimoto N. An animal model of cerebral aneurysms. Neuropathol Appl Neurobiol 1987;13:77-90

45. Yamazoe N, Hashimoto N, Kikuchi H, et al. Elastic skeleton of intracranial cerebral aneurysms in rats. Stroke 1990;21:1722-26

46. Cawley CM, Dawson RC, Shengelaia G, et al. Arterial saccular aneurysm model in the rabbit. AJNR Am J Neuroradiol 1996;17:1761-66

47. Costalat V, Sanchez M, Ambard D, et al. Biomechanical wall properties of human intracranial aneurysms resected following surgical clipping (IRRAs Project). J Biomech 2011;44:2685-91

48. Steiger HJ, Aaslid R, Keller S, et al. Strength, elasticity and viscoelastic properties of cerebral aneurysms. Heart Vessels 1989;5:41-46

49. Aoki T, Kataoka H, Moriwaki T, et al. Role of TIMP-1 and TIMP-2 in the progression of cerebral aneurysms. Stroke 2007;38:2337-45

50. Aoki T, Kataoka H, Morimoto M, et al. Macrophage-derived matrix metalloproteinase- 2 and -9 promote the progression of cerebral aneurysms in rats. Stroke 2007;38:162-69

51. Ishibashi R, Aoki T, Nishimura M, et al. Contribution of mast cells to cerebral aneurysm formation. Curr Neurovasc Res 2010;7:113-24

52. Killer-Oberpfalzer M, Aichholzer M, Weis S, et al. Histological analysis of clipped human intracranial aneurysms and parent arteries with short-term follow-up. Cardiovasc Pathol 2012;21:299-306

53. Kosierkiewicz TA, Factor SM, Dickson DW. Immunocytochemical studies of atherosclerotic lesions of cerebral berry aneurysms. J Neuropathol Exp Neurol 1994;53:399-406

54. Coen M, Burkhardt K, Bijlenga P, et al. Smooth muscle cells of human intracranial aneurysms assume phenotypic features similar to those of the atherosclerotic plaque. Cardiovasc Pathol 2013;22:339-44

55. Ross R. Atherosclerosis-an inflammatory disease. $N$ Engl J Med 1999;340:115-26

56. Dessi M, Noce A, Bertucci P, et al. Atherosclerosis, dyslipidemia, and inflammation: the significant role of polyunsaturated fatty acids. ISRN Inflammation 2013; http://dx.doi.org/10.1155/2013/ 191823. Accessed October 3, 2013

57. Krychtiuk KA, Kastl SP, Speidl WS, et al. Inflammation and coagulation in atherosclerosis. Hamostaseologie 2013;33:269-82

58. Gaetani P, Tartara F, Grazioli V, et al. Collagen cross-linkage, elastolytic and collagenolytic activities in cerebral aneurysms: a preliminary investigation. Life Sci 1998;63:285-92

59. Umeda Y, Ishida F, Hamada K, et al. Novel dynamic four-dimensional CT angiography revealing 2-type motions of cerebral arteries. Stroke 2011;42:815-18

60. Nishida T, Kinoshita M, Tanaka H, et al. Quantification of cerebral artery motion during the cardiac cycle. AJNR Am J Neuroradiol 2011;32:E206-08

61. Boecher-Schwarz HG, Ringel K, Kopacz L, et al. Ex vivo study of the physical effect of coils on pressure and flow dynamics in experimental aneurysms. AJNR Am J Neuroradiol 2000;21:1532-36

62. Ueno J, Matsuo T, Sugiyama K, et al. Mechanism underlying the prevention of aneurismal rupture by coil embolization. J Med Dent Sci 2002;49:135-41
63. Mitha AP, Reichardt B, Grasruck M, et al. Dynamic imaging of a model of intracranial saccular aneurysms using ultra-high-resolution flat-panel volumetric computed tomography: laboratory investigation. J Neurosurg 2009;111:947-57

64. Gupta R, Cheung AC, Bartling SH, et al. Flat-panel volume CT: fundamental principles, technology, and applications. Radiographics 2008;28:2009-22

65. Topcuoglu MA. Transcranial Doppler ultrasound in neurovascular diseases: diagnostic and therapeutic aspects. J Neurochem 2012; 123(suppl 2):39-51

66. $\mathrm{Ng} \mathrm{A}$, Swanevelder J. Resolution in ultrasound imaging. Contin Educ Anaesth Crit Care Pain 2011;11:186-92

67. Barlinn K, Zivanovic Z, Zhao L, et al. Intracranial vessel localization with power motion Doppler (PMD-TCD) compared with CT angiography in patients with acute ischaemic stroke. Int J Stroke 2013;8:398-402

68. Weishaupt D, Koechli VD, Marincek B. How does MRI work? An Introduction to the Physics and Function of Magnetic Resonance Imaging. 2nd ed. Berlin: Springer-Verlag; 2006:78-81

69. Tsuruda JS, Halbach VV, Higashida RT, et al. MR evaluation of large intracranial aneurysms using cine low flip angle gradient-refocused imaging. AJR Am J Roentgenol 1988;151:153-62

70. Yan L, Wang S, Zhuo Y, et al. Unenhanced dynamic MR angiography: high spatial and temporal resolution by using true FISP-based spin tagging with alternating radiofrequency. Radiology 2010;256:270-79

71. Gerretsen SC, Versluis B, Bekkers SC, et al. Cardiac cine MRI: comparison of 1.5 T, non-enhanced 3.0 $\mathrm{T}$ and blood pool enhanced 3.0 $\mathrm{T}$ imaging. Eur J Radiol 2008;65:80-85

72. Hadizadeh DR, Marx C, Gieseke J, et al. High temporal and high spatial resolution MR angiography (4D-MRA). Rofo 2014;186:847-59

73. Davis B, Royalty K, Kowarschik M, et al. 4D digital subtraction angiography: implementation and demonstration of feasibility. AJNR Am J Neuroradiol 2013;34:1914-21

74. Pontone G, Bertella E, Mushtaq S, et al. Coronary artery disease: diagnostic accuracy of CT coronary angiography - a comparison of high and standard spatial resolution scanning. Radiology 2014;271:688-94

75. Sabarudin A, Sun Z. Coronary CT angiography: diagnostic value and clinical challenges. World J Cardiol 2013;5:473-83

76. Sepehri S, Zouaoui K, Thiran J-P. A realistic computed tomography simulator for small motion analysis of cerebral aneurysms. In: Proceedings of the 35th Annual International Conference of the IEEE Engineering in Medicine and Biology Society, Osaka, Japan. July 3-7, 2013

77. Thiran JP, Sepehri S. Direct detection of small motion from dynamic tomography images. In: Proceedings of the 21st European Signal Processing Conference, Marrakech, Morocco. September 9-13, 2013

78. Mathews JD, Forsythe AV, Brady Z, et al. Cancer risk in 680,000 people exposed to computed tomography scans in childhood or adolescence: data linkage study of 11 million Australians. BMJ 2013;346:f2360 